\title{
Le jeu en contexte scolaire : un outil au service du renouveau didactique de la géographie?
}

Frédérique Jacob et Emilie Servais

\section{(2) OpenEdition}

1 Journals

Édition électronique

URL : https://journals.openedition.org/sdj/880

DOI : $10.4000 /$ sdj. 880

ISSN : 2269-2657

\section{Éditeur}

Laboratoire EXPERICE - Centre de Recherche Interuniversitaire Expérience Ressources Culturelles Education

\section{Référence électronique}

Frédérique Jacob et Emilie Servais, «Le jeu en contexte scolaire : un outil au service du renouveau didactique de la géographie? ", Sciences du jeu [En ligne], 8 | 2017, mis en ligne le 27 décembre 2017, consulté le 15 septembre 2021. URL : http://journals.openedition.org/sdj/880 ; DOI : https://doi.org/ $10.4000 /$ sdj. 880

Ce document a été généré automatiquement le 15 septembre 2021.

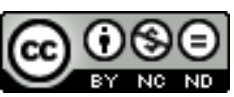

La revue Sciences du jeu est mise à disposition selon les termes de la Licence Creative Commons Attribution - Pas d'Utilisation Commerciale - Pas de Modification 4.0 International. 


\title{
Le jeu en contexte scolaire : un outil au service du renouveau didactique de la géographie?
}

\author{
Frédérique Jacob et Emilie Servais
}

1 Si jouer pour apprendre n'est pas un choix récent, dans l'imaginaire collectif, l'ambigüité entre le travail utile et le jeu futile, initiée dès l'Antiquité, renforcée au Moyen Age par la culture chrétienne, perdure encore de nos jours (Brougère, 1995). Comme le souligne V. Potier (2016), le jeu sérieux d'apprentissage se caractérise par sa légitimité institutionnelle grandissante. Ainsi, le programme de cycle $1^{1}$ préconise une école qui organise des modalités spécifiques d'apprentissage en insistant sur la mise en œuvre de situations d'apprentissage telles que le jeu (apprendre en jouant), la résolution de problèmes (apprendre en réfléchissant et résolvant des problèmes), des entrainements, etc. Par ailleurs, les ressources pédagogiques destinées aux enseignants, disponibles sur les sites gouvernementaux, sont nombreuses, telles apprendre avec le jeu numérique ${ }^{2}$ ou encore sur les sites des circonscriptions.

2 C'est sur ce recours au jeu dans la discipline scolaire géographie que nous souhaitons nous interroger. Nous voulons voir dans la ludification des activités de la classe une volonté de renouveau des objectifs didactiques et non une mode. Nous souscrivons aux travaux les plus récents des didacticiens de la géographie (Pache, Hertig, Curnier, 2016) qui démontrent que de nouvelles exigences dans les domaines du savoir et des compétences notamment par l'introduction dans les curricula du développement durable, de la citoyenneté, etc. entrainent une recomposition de la discipline. Ainsi, la géographie enseignée se tourne vers le monde social et la perspective actancielle. Faire jouer devrait donc ambitionner de renouveler les finalités de la discipline. Il nous parait donc important que les enseignants ne choisissent pas de recourir au jeu seulement pour motiver des élèves mais bien, pour faciliter la prise de conscience que la géographie est une discipline qui offre des clés de compréhension du monde. Comment faciliter l'utilisation des jeux chez les enseignants dans des séquences 
d'enseignement-apprentissages pour qu'ils développent des savoirs et des compétences renouvelées?

3 Aussi, notre questionnement central est le suivant : serait-il possible de mettre à leur disposition un outil d'aide à la réflexion qui faciliterait leurs choix de démarches. Par le jeu, la géographie enseignée pourrait-elle (re)trouver du sens auprès des élèves, en privilégiant la compréhension des enjeux territoriaux, objectif primordial de cette discipline? $\mathrm{Si}$, pendant plus des trois-quarts $\mathrm{du} \mathrm{XX}^{\mathrm{e}}$ siècle, il était important de connaitre les lieux pour connaitre les hommes et de fait, le fait d'apprendre par cœur des capitales, des préfectures avait un sens, il n'en est plus rien aujourd'hui. En revanche, être conscient de son et de ses actions dans l'organisation d'un territoire, des interactions et de leurs conséquences avec les autres est épistémologiquement valable et didactiquement utile. Ainsi, l'observation, la classification, la mémorisation, si attachées aux représentations de la discipline, pourraient-elles laisser leur place à des démarches favorisant le raisonnement, la construction de modèles, l'éveil de la responsabilité, l'initiative, etc?

Notre propos n'est pas de définir le jeu que d'autres ont tenté avant nous, ni de montrer la réalité des usages du jeu dans les classes. Nous proposons ici de dégager pas à pas les éléments de notre démarche permettant l'élaboration d'un outil d'aide à la réflexion. Tout d'abord, l'élaboration d'une grille d'analyse, à partir d'un premier ensemble de jeux, accessibles sur le site réseau Ludus: jouer en classe-apprendre avec les jeux ${ }^{3}$ qui permet de repérer les intentions pédagogiques et didactiques des enseignants de façon à prendre la mesure du sens qu'accordent, donnent ou croient donner les enseignants à la pratique du jeu en classe. Cette grille (annexe) permet la collecte ordonnée des informations présentes dans les fiches de présentation des jeux du site. La seconde phase permet la production d'une classification puis d'une typologie de jeux. Pour nous, au sommet de la typologie, le jeu T'es ou cubo ? prend le statut de jeumodèle : c'est en effet un jeu d'aménagement spatial, qui offre aux élèves la possibilité d'agir et surtout de comprendre les règles d'organisation de l'espace. Ce n'est qu'avec des jeux de ce type, que les enseignants de géographie pourront mener à bien leurs objectifs spécifiques à savoir : manipuler des notions, apprendre à raisonner, etc.

\section{Jeu en classe : petits moyens, grands effets ?}

5 Nous allons initier notre démarche par l'analyse à plat de la grille (annexe), ceci pour analyser les intentions des enseignants lorsqu'ils décident de créer et de recourir au jeu avec leurs élèves et repérer si le recours à une pratique pédagogique alternative (ou innovante) sous-tend un discours géographique renouvelé.

Notre choix de jeux est guidé par l'existence du site du réseau Ludus qui parait être le plus proche de nos interrogations. Porté par des enseignants depuis de nombreuses années (près de 20 ans), il a pour objectif d'assurer la promotion, la mutualisation et l'innovation. Le site est toujours actif même si certains jeux commencent à dater. Nous y ajoutons le jeu T'es ou cubo? qui offre l'opportunité d'être pensé pour des élèves d'école élémentaire (cycle 2) et à ce titre complémentaire des vingt autres jeux, pensés pour le secondaire. Il a été choisi car expérimenté dans un mémoire de Master 2, par Emilie Servais, le déroulé complet du jeu est connu. Pour explorer l'ensemble des jeux, la grille (en annexe) a facilité une collecte des informations assez larges et présentes dans les fiches de présentation des jeux. Ainsi, vingt critères ont été pris en compte : le 
nom et le prénom des concepteurs, l'intitulé du jeu ou le niveau de classe, le nombre de joueurs, le coût et la durée du jeu qui permettent un premier contact avec le jeu, la nature du jeu (adaptation ou création), le type (jeu de société, jeu de rôle, de simulation, etc.), le matériel utilisé, le déroulé, les objectifs annoncés, l'existence de gagnants (ou pas); autant de critères qui offrent l'opportunité de cibler le jeu, de le référencer à des jeux existants. La façon dont sont construits les savoirs (quand il y en a), les règles du jeu, le rôle de l'enseignant, celui du hasard lors de la phase du jeu permettent de concevoir peu ou prou la géographie pratiquée. Dans ce cadre, le moment de la séance où le jeu est convoqué, la démarche, le rôle de l'espace (virtuel, connu, vécu), la volonté de faire raisonner les élèves ont fait l'objet d'une observation attentive. La date de création aurait été une information précieuse, mais elle n'est pas accessible sur le site (la date proposée est souvent celle de la réactualisation du site). Chaque jeu a été passé au crible de la grille, et permet de répondre aux interrogations suivantes.

7 La première interrogation a porté sur le genre des auteurs : proposer le jeu en classe est-il affaire de genre ? Sur les 19 jeux analysés, si l'un n'a pas d'auteur (le lien proposé vers la revue TDC n'est plus actif) et un autre est créé par Dominique N. (prénom indifférencié), la parité est parfaitement respectée : 6 auteures qui produisent 8 jeux et 6 auteurs qui produisent 9 jeux. 2 femmes et 2 hommes produisent plusieurs jeux chacun. Si nous ne pouvons connaître les motivations, l'âge, l'ancienneté dans la carrière enseignante, nous pouvons signaler que le recours au jeu ne serait donc pas réservé à un genre. Si cette information peut paraitre hors sujet, elle viendrait enrichir un questionnement ultérieur sur les motivations des enseignants pour le jeu et l'innovation. Il ne s'agit là que d'une indication.

8 Le deuxième point, pour lequel nous avons dû extrapoler un peu, car les données manquent sur le site, est le coût du jeu à l'école. Utiliser le jeu en classe en génère-t-il un? A la lecture du matériel nécessaire pour mener les jeux en classe, nous pouvons constater que le plus fréquemment, des feuilles A3, des plateaux cartonnés et des documents plastifiés sont la base du jeu à laquelle s'ajoutent des dés, des pions. Proposer un jeu à l'école est un bricolage, dans le sens premier du terme, c'est-à-dire qui nécessite d'exécuter, pour les enseignants, de petits travaux qui réclament de l'ingéniosité et de l'habileté manuelle et qui ne nécessitent aucun investissement financier. La limite serait lorsqu'il s'agit de jeu en ligne (Simcity ou autres) où l'achat du logiciel ou de la licence peut engendrer des frais qui devraient alors être supportés par le budget de l'établissement. L'investissement majeur est le temps, hors de la classe, que les enseignants accordent à la création du jeu. Ainsi, la jeune fonctionnaire stagiaire, inventrice du jeu T'es où cubo ?, a dû rencontrer les responsables de la gestion de la propreté de la commune où se trouve son lieu de stage, collecter les informations concernant les PAV (points d'apports volontaires), les repérer, les localiser sur une carte de la commune afin de constituer le document de référence. Pour le reste, dans ce cas aussi, des photocopies en format A3 et A4 ainsi que des gommettes de couleur (rouges et vertes) ont été nécessaires, rien de plus.

9 Le troisième point est la durée du jeu. Mettre en œuvre un jeu nécessite-t-il de modifier l'organisation temporelle de la classe ? Dans la quasi-totalité des jeux analysés, la durée est celle d'une séance (soit 55'). Le jeu se calque sur les pratiques usuelles pour ne pas impliquer une modification d'emploi du temps. Les jeux se déroulent dans la salle de classe habituelle (voire une salle pupitre/informatique) où seule l'organisation spatiale 
est sans doute repensée (notamment pour les jeux en équipe où l'îlot paraît le plus judicieux). Tous les jeux du site (18) ne connaissent qu'une seule phase plus ou moins longue (de 15' pour Ludoville à 2 heures pour Le jeu de la diversité européenne) et en début de séquence ( 7 jeux) ou en fin ( 3 jeux). Certains jeux (4) sont indépendants de la séquence et peuvent se jouer n'importe quand. Pour tous les autres (4), les auteurs n'ont pas précisé le moment où ils pensent qu'il est le plus judicieux de jouer. T'es où cubo ? se joue tout au long de la séquence, en deux ou trois phases. Une progression est pensée, notamment pour les notions travaillées, le changement d'échelle, la complexité de la tâche et le lien avec la problématique de la séquence.

Le quatrième point s'intéresse au niveau de classe pour lequel est proposé le jeu. L'activité «jeu » est-elle réservée aux enfants les plus jeunes? Le réseau Ludus est animé et organisé par et pour des enseignants du second cycle. La quasi-totalité des jeux sont prévus pour le collège, trois le sont pour le lycée (classe de $2^{\text {de }}$ ). 7 des 18 jeux sont pensés pour des classes de $6^{\text {e }}$. Même si l'utilisation du jeu se généralise, les classes " sérieuses » (Première et Terminale) sont très peu concernées. Lors de nos recherches, nous n'avons pas pour le moment repéré de jeux pour ces deux niveaux de classe, cela ne veut pas dire qu'ils n'existent pas, mais tout du moins qu'ils ne sont pas diffusés.

11 Le cinquième point porte sur le nom attribué au jeu. Le choix du nom par le concepteur est-il révélateur des objectifs premiers, pédagogiques ou didactiques? La très grande banalité des noms proposés montre qu'aucun effort de marketing n'est fait : le jeu n'est pas prévu pour être commercialisé, tout juste pour être mutualisé, au mieux diffusé au sein de l'Education nationale. Une première interrogation porte sur l'impact du nom auprès des élèves. Il ne nous est pas possible de savoir si le nom du jeu est communiqué aux élèves ou pas. Mais, si les intentions portent sur la motivation, celle-ci devrait passer en premier lieu par l'annonce du nom. Si Grenouille-autoroute, Happywood, ou encore Littoland peuvent créer une émulation chez les joueurs, Benidorm, Transition démographique, Régions françaises n'offrent pas beaucoup de suspens sur le contenu du jeu.

12 Le dernier point de ce premier faisceau d'analyse est induit du précédent et porte sur la dénomination donnée par l'enseignant aux élèves, lors de la présentation de l'activité. Si l'auteur reconnaît bien qu'il fabrique un jeu, puisqu'il le dépose sur le site, annonce$\mathrm{t}$-il et utilise-t-il le terme jeu face aux élèves dans la classe ? On peut en douter à l'étude du nom de certains jeux et par l'expérience menée par E. Servais; en classe, le terme de jeu n'est jamais prononcé. Elle n'a pas osé dire à ses élèves qu'ils allaient jouer : peur de soucis de gestion de classe, de légèreté de l'implication des élèves, du regard des parents, des collègues voire de la conseillère pédagogique. Le nom T'es où cubo ? est donné, a posteriori, dans le cadre de l'écriture d'articles avec une volonté d'interpeler les lecteurs: titre court, à la limite de l'onomatopée, le tutoiement donnant une proximité avec le conteneur de tri sélectif pour le verre, objet peu motivant s'il en est. Ce choix est dans la lignée du titre du jeu référence (Jacob, 2005) GPS - géographic poubelle situation. Concernant les 18 jeux, 5 ont des noms créés par leurs auteurs (Ludoville, Littoland, Géotrouvetout, Happywood et Autoroute grenouille) et 6 reprennent l'intitulé jeu (Jeu du Maghreb, Les 25 familles d'Europe, Jeu de la diversité européenne, Jeu de l'Amérique, Cartopuzzle, La course pour la carte). Les autres (6) ont des intitulés géographiques (Transition démographique, Benidorm, Milieux naturels, DD en Inde, Littoral).

13 Ce constat initial montre que le jeu ne semble pas toujours modifier les pratiques usuelles. Nous ne pouvons qu'émettre l'hypothèse qu'un éventail de démarches 
pourrait dépendre de la personnalité, de l'environnement professionnel et de l'ancienneté dans la carrière des concepteurs. Les fiches disponibles sur le site réseau Ludus ne nous en disent guère plus sur les motivations des concepteurs de jeu. Néanmoins, nous repérons bien des enseignants en tension (Fontanabona, Thémines, 2005) entre les conceptions qu'ils se font de l'enseignement en général (intérêt pour la réussite des élèves, recherche de la motivation, etc.) et de l'enseignement de la géographie en particulier (discipline de l'observation, de la classification, de la mémorisation, ou discipline du raisonnement). Le jeu questionne donc le pourquoi de son utilisation en classe. La grille ne nous permet pas de savoir dans quelle mesure, les élèves jouent-ils? Et qu'apprennent-ils réellement? C'est là un autre volet de nos recherches qui reste à entreprendre en convoquant non plus des fiches descriptives de jeu mais les principaux intéressés : les élèves.

\section{Enseigner la géographie par le jeu : une expérience spatiale renouvelée}

14 Les références sont nombreuses au tournant des années 1990/2000 pour évoquer une géographie enseignée en crise. S. Rimbert (1998) propose même de supprimer la géographie, discipline ennuyeuse, enseignée par des historiens. Les propositions des didacticiens (Merenne-Schoumaker, 2005, Masson Vincent, 2004) ont un faisceau d'objectifs communs : rendre la discipline opératoire, utile socialement; la géographie devrait permettre aux élèves de comprendre les enjeux territoriaux, pour devenir des acteurs réflexifs de leurs territoires. Avec J.F. Thémines (2004), nous pensons que les élèves doivent comprendre que les productions spatiales sont le résultat d'interactions complexes et systémiques entre des facteurs plus ou moins nombreux, dont celles construites entre individus. D'idiographique, la géographie enseignée deviendrait nomothétique et surtout complexe, systémique et même teintée d'incertitude. Les nouveaux programmes du primaire et collège $\mathrm{e}^{4}$, analysés par des contributeurs $d u$ blogue didagé $0^{5}$, mettent le raisonnement au cœur des apprentissages en histoire géographie au cycle 3, par le biais de la compétence « raisonner, justifier une démarche et les choix effectués »: poser des questions, se poser des questions, formuler des hypothèses, vérifier, justifier. C'est une profonde actualisation épistémologique et didactique et la fin des programmes centrés sur des contenus à enseigner. L'encyclopédisme, la classification et la mémorisation laissent la place à l'initiation à raisonner. M. Masson-Vincent (1994) prônait déjà une initiation au raisonnement géographique par le recours à des questions simples, « Qui ? Fait quoi ? Où ? Pourquoi ? Comment?" , auxquelles, il serait possible d'ajouter par qui ? Pour qui ? Pourquoi là et pas ailleurs? A. Le Roux (2003) insistait sur l'importance de penser les programmes en termes de concept et notion plus qu'évènement factuel et localisation. Dans les deux cas, l'idée est bien d'organiser, de structurer les savoirs pour faciliter les apprentissages.

15 Les jeux du réseau Ludus sont-ils révélateurs de ces glissements épistémologiques et didactiques? Malgré le petit nombre d'exemples retenus, nous avons mis en place une classification de ces jeux. C'est la deuxième phase de l'analyse. La figure 1 permet d'explorer selon deux axes les jeux à l'aune des critères épistémologiques et didactiques. Le premier axe propose une gradation des choix didactiques entre deux positions relevant de deux approches épistémologiques opposées que nous qualifions 
de " géographie post-vidalienne » et " approche rénovée de la géographie enseignée ». Et le second axe suggère une gradation de choix didactiques mais relevant de pratiques d'élèves dans la construction citoyenne.

Figure 1 : classification des jeux du réseau Ludus au regard des évolutions didactiques de la géographie enseignée

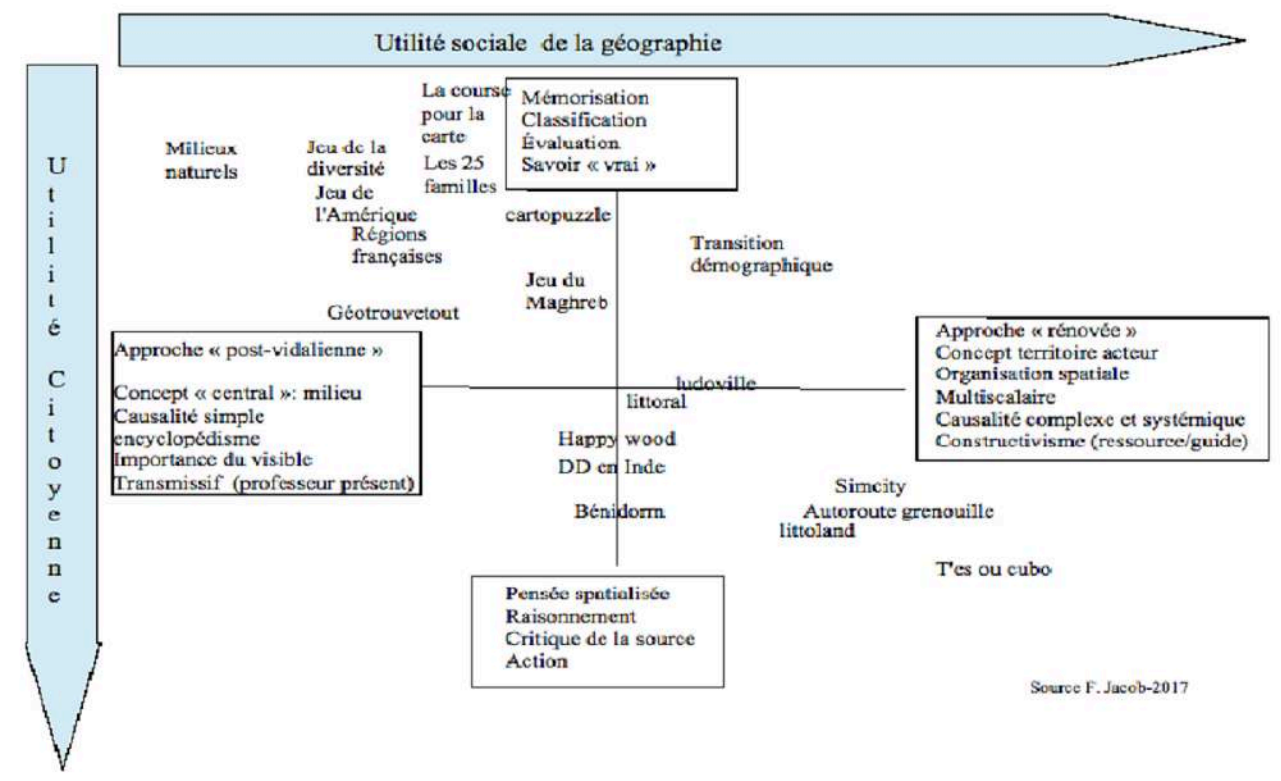

L'axe 1 permet de classer les jeux en fonction des choix conceptuels de causalité : d'une causalité simple à une causalité complexe et systémique, du rôle de l'enseignant en fonction du rôle qu'il s'est attribué dans le jeu de très présent (arbitre, meneur de jeu, etc.) à absent lors du déroulement du jeu. Ainsi, prenons l'exemple du jeu Milieux naturels. Du point de vue didactique, l'auteur fait le choix d'aborder le concept de milieu, concept «classique » s'il en est. L'objectif est de repérer les caractéristiques d'un milieu naturel. Là encore, l'approche n'est guère rénovée. L'étude est celle d'un tableau plus que d'un processus. Du point de vue pédagogique et cognitif, l'objectif est la mémorisation des climats par la reconduction du jeu. L'approche behavioriste semble favorisée. La démarche proposée nous paraît être très proche d'une approche "post-vidalienne ", à l'inverse de Littoland. Ce jeu propose aux élèves de comprendre l'aménagement d'un littoral virtuel, sans recourir au hasard (ni dé, ni carte piège, etc.). La prise en compte de facteurs explicatifs multiples, qui interagissent entre eux, comme d'autres jeux de simulation, serait proche d'une démarche qualifiée de rénovée. T'es où cubo ? se déroule sur le territoire connu des élèves (la classe, le quartier, la commune), propose une démarche multi-scalaire, définit clairement les notions travaillées (flux et mailles) et permet aux élèves de comprendre que les Points d'Apport Volontaire ne sont pas placés au hasard dans la ville, mais qu'ils répondent à des règles d'organisation; il est donc le plus à droite de l'axe 1 .

L'axe 2 organise les compétences du moins vers le plus d'utilité citoyenne. La course pour la carte est inspirée du Jeu des 1000 Bornes, les élèves ont pour objectif de rejoindre un point le plus rapidement possible, lorsque les adversaires doivent les en empêcher. Le hasard a une très grande place (dé, tirage au sort de carte, espace virtuel), les élèves s'amusent, certes, mais quels sont les apprentissages? Le jeu est donc situé en haut de l'axe, où les compétences citoyennes sont peu pensées. A l'autre extrémité de l'axe, 
Benidorm ou Littoland proposent d'aménager des littoraux, de repérer les jeux d'acteurs, de se poser des questions, de soumettre des choix. Ils sont bien en situation de construire des savoirs, de prendre en compte la complexité d'un territoire. La classification ainsi constituée nous permet d'envisager une typologie des jeux. La préfiguration que nous proposons repose sur la distinction de 4 types de jeu (figure 2).

Figure 2 : Les quatre types de jeux du réseau Ludus

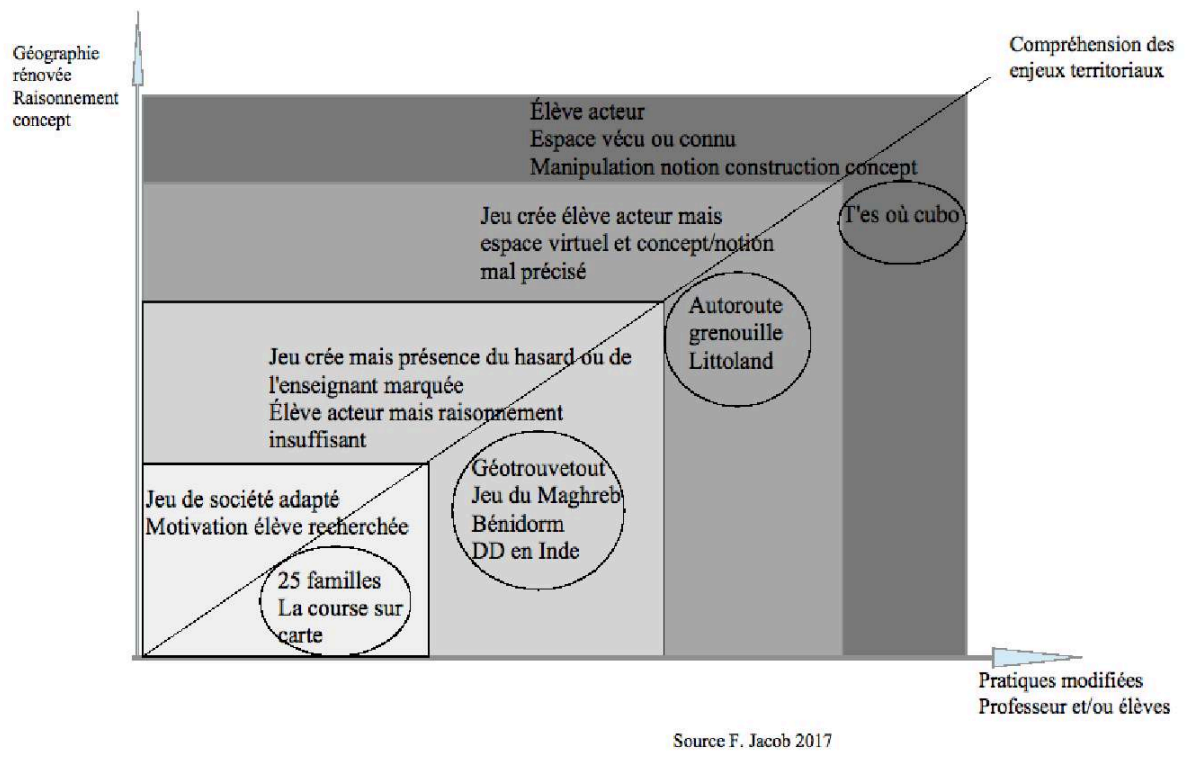

Il n'est pas aisé de définir une typologie du jeu, en géographie comme en tout. Nous nous y essayons en connaissant la part de subjectivité dont elle résulte, du manque d'exhaustivité de nos références, du potentiel à exploiter et à développer.

Le premier type de jeu est l'adaptation de jeux de société (puzzle, jeu des 7 familles, jeu de l'Oie, Trivial Pursuit, etc.). Régions françaises, La course sur la carte, Les 25 familles reflètent la vision la plus traditionnelle de l'enseignement de la géographie (encyclopédisme, classification, mémorisation). Ils n'apportent pas un grand intérêt didactique pour le renouvellement de l'enseignement de la géographie. Ils offrent peutêtre plus l'envie de mémoriser des notions, des repères auprès d'élèves plus ou moins motivés.

Le second type de jeu est une re-création simplifiée de l'espace par l'enseignant, en fait une nouvelle activité destinée aux élèves. Contrairement au cours magistral, voire dialogué, l'objectif de l'enseignant est de proposer une situation d'apprentissage où l'élève est bien en situation de construire ses apprentissages. La motivation peut être aussi un plus. Pourtant, le rôle de l'enseignant est prégnant, le hasard (dé, carte à jouer, etc.) ne permet pas la compréhension des interactions entre les facteurs explicatifs. Ainsi, le Jeu du Maghreb propose de repérer les caractéristiques des trois pays, et de localiser des types d'espace. Pour ce faire, de nombreuses photographies (16) sont le support du jeu comme voulant balayer la totalité des types d'espace. Mais, il n'est pas possible d'envisager un tel catalogue et de viser l'exhaustivité : l'enseignant ne propose dès lors que ses propres représentations. De plus, la part belle est faite au visible par l'analyse de paysages. C'est là un risque important de vouloir transcrire un réel complexe dans un réel virtuel, simplifié voire caricatural. De même, l'impact du hasard 
ne risque-t-il pas d'être associé à un facteur explicatif (créant une forme de déterminisme : que peut-on faire contre le hasard ?). Enfin, même lorsque la notion travaillée est clairement annoncée (comme la transition démographique ou DD en Inde), la simplification, due à la «jouabilité " voire aux règles du jeu empêche une approche complexe et le raisonnement.

Le troisième type de jeu repéré évoque une volonté de simulation. L'espace-support à étudier est créé par l'enseignant. Cette approche virtuelle (une feuille quadrillée pour Autoroute-grenouille ou pour Littoland) s'inscrit dans l'idée que l'espace est un support neutre. Pour respecter une cohérence épistémologique, le jeu devrait privilégier une approche hypothético-déductive, s'appuyant sur un modèle théorique, développant des hypothèses à vérifier, pour valider ou enrichir le modèle initial. Les jeux précités sont construits sur une démarche inductive, après une première phase d'observation (la réalisation $\mathrm{du} \mathrm{jeu}$ ), une généralisation est menée. L'incohérence de deux angles si opposés rend impossible une démarche scientifique valable.

Le quatrième type de jeu convoque l'émulation. Le jeu T'es où cubo? propose de travailler sur l'espace connu des élèves (territoire). C'est le seul jeu de notre panel qui fait ce choix et qui, de plus, est multiscalaire et annonce clairement les notions manipulées (flux et maille). L'enseignant est bien en situation de fournir à l'élève une expérience spatiale au cours de laquelle celui-ci est amené à raisonner et comprendre le monde dans lequel il vit. L'objectif est de faire repérer les conflits d'usage ou d'acteurs, d'en comprendre les choix et de donner du sens aux actes de chacun. Ainsi, tout projet d'aménagement peut être un support à un jeu spatial (jeu de rôle ou jeu d'aménagement) : la création d'une bretelle d'autoroute, l'installation d'un commerce, le changement de fonction d'un quartier, etc.

\section{« T'es où cubo ? » un jeu d'aménagement spatial transposable}

Nous proposons de décrire les phases du jeu, ses objectifs et son déroulé car nous le savons déclinable selon l'espace proche et l'âge des élèves. Le jeu a été proposé à une classe de CE1, d'une école élémentaire constituée de cinq classes, d'une petite ville (17 267 habitants) située près de Hénin-Beaumont, dans le Pas-de-Calais. Cette commune jeune (30\% de la population a moins de vingt ans) dispose de sept écoles. L'une d'entre elles, support de l'expérimentation, accueille des enfants issus d'une forte diversité sociale et culturelle. $T^{\prime \prime} e s$ où cubo ? s'intègre dans une séquence de quatre heures. Dans une première séance, sur un plan de la classe, en travail individuel, les élèves sont amenés à positionner la poubelle de la classe à une place qui selon eux, serait la plus pratique. Pour ce faire, les élèves ont chacun une gommette de couleur et un plan de la classe. L'objectif est de permettre à l'élève de prendre une décision et de la justifier. L'élève répond à la question première du raisonnement géographique « où ?»: où je mets où la poubelle? Puis il répond à une deuxième interrogation " pourquoi ? » et peut-être même " pourquoi ici/là et pas ailleurs? ». Concernant les 25 élèves de CE1, 11 élèves positionnent leur poubelle en fonction du bureau (le leur ou celui de l'enseignante) et 12 hors de leur portée, soit à proximité de la porte ou du tableau (deux font un choix autre non expliqué : il s'agit de deux élèves en situation de handicap accompagnés par une AVS, pour qui on peut émettre l'hypothèse qu'une seule séance de jeu ne permet pas de s'adapter à cette nouvelle forme d'activité). Plus de $90 \%$ 
des élèves positionnent leur poubelle implicitement selon la maille ou le flux, choix partagé à égalité.

Figure 3 : Un plan d'élève

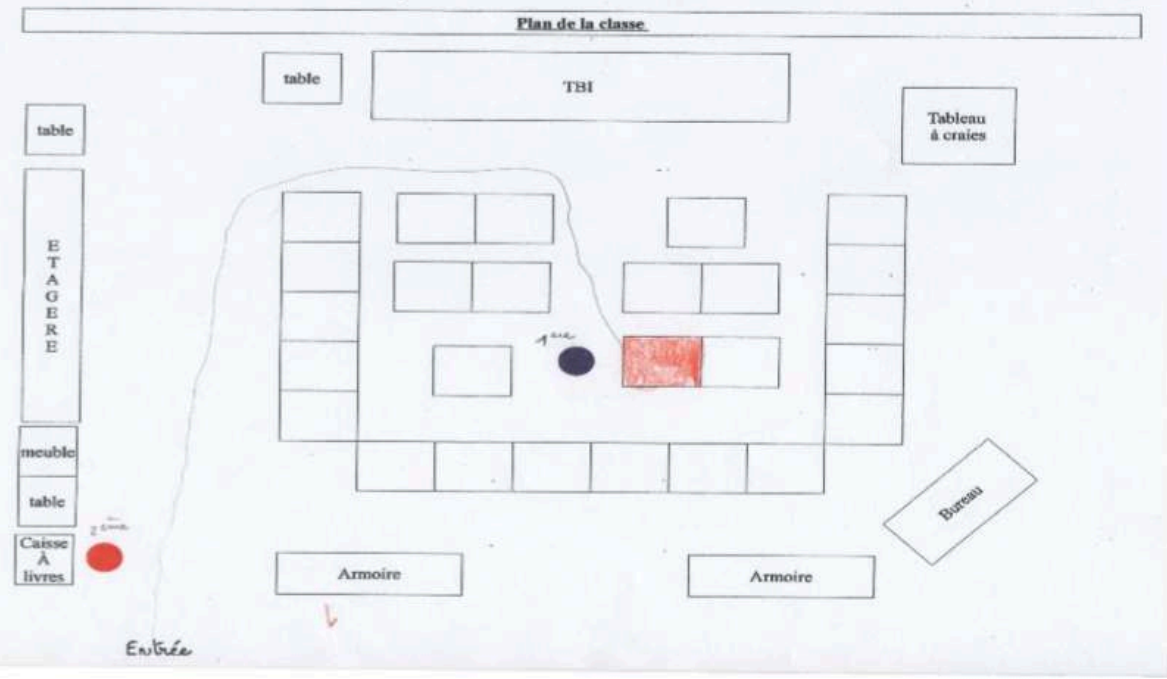

Localisation de sa place et représentation initiale de la situation appropriée de la poubelle selon lui gommette noire (7êre), trajet de l'élève (Servais 2015)

La mise en commun est effectuée au tableau blanc interactif. Chaque élève de la classe vient tracer le trajet qu'il suit pour se rendre à sa place depuis la porte d'entrée. Cet ajout de consigne permet de visualiser le lieu de passage obligé des élèves qui passent tous par la porte puis ensuite se dirigent de façon individuelle vers leur table.

Figure 4 : tracé du trajet des élèves de l'entrée à leur place au TBI (Servais 2015)

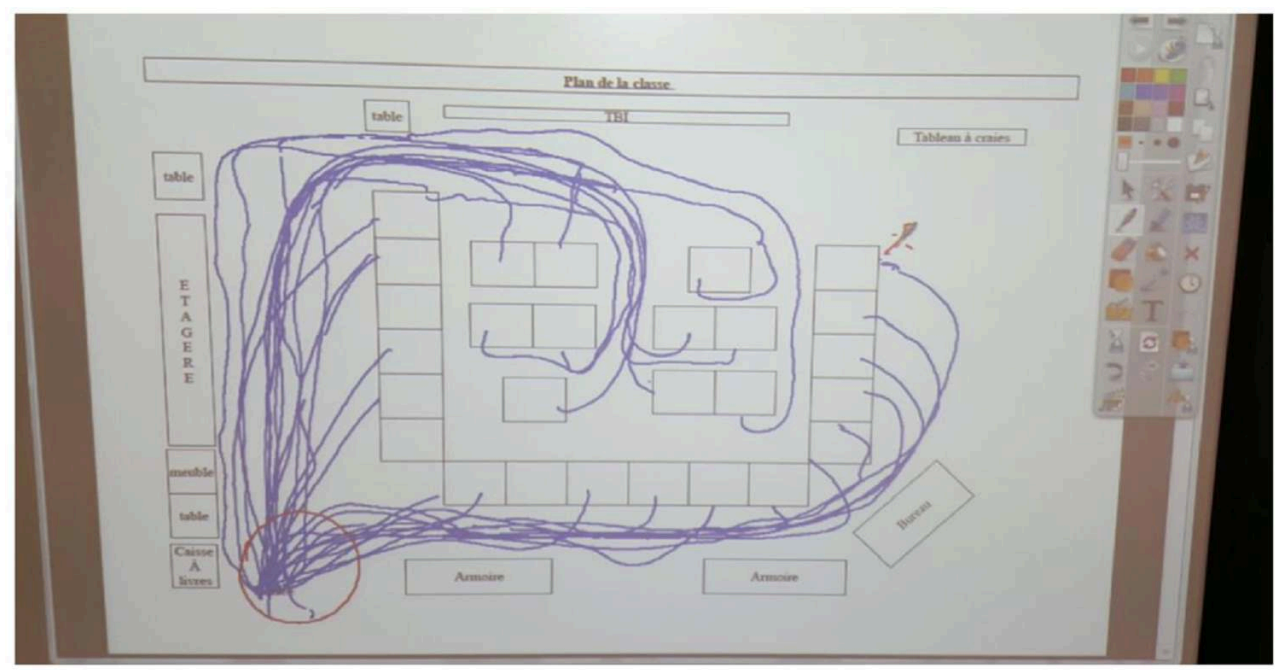

Lors de la mise en commun, l'enseignante fait repérer qu'il existe deux localisations récurrentes de la poubelle : près de la porte, là où tous les élèves passent - rond rouge sur la figure 3 - (flux) ou à portée de bras, soit la poubelle à proximité de la table (maille). Nous comprenons ces deux notions selon une approche quantitativiste: la 
maille est l'élément de base, révélatrice du découpage de l'espace qui permet son appropriation, sa gestion ou sa connaissance. Le maillage relève de la figure de l'aire l'espace qu'il découpe - et du réseau, formé par les limites de ce maillage. Le flux est l'écoulement, le transfert, d'une certaine quantité de personnes, de véhicules, d'informations, de marchandises, transportés par un moyen de communication, par le biais d'un réseau. Les poubelles ne sont pas disposées au hasard, mais que des règles spatiales justifient leur localisation. Il est à noter que jamais les mots de flux et de maille ne sont prononcés par l'enseignante.

Lors de la deuxième séance, dont la durée est encore d'une heure, sur un extrait d'un plan simple et quadrillé de la ville (type plan du calendrier des postes ou plan touristique), il est demandé aux élèves lors d'une phase de travail individuel de positionner les sept cubo'verres sur le plan de la ville. L'enseignante projette sur le quartier de l'école le raisonnement géographique construit dans la classe. Où positionner les cubo'verres? Pourquoi ? Et maintenant, pour qui ? Elle fait l'hypothèse que le changement d'échelle, la prise en main d'un nouveau plan ne seront pas des problèmes pour les élèves. Effectivement, le changement d'échelle ne semble pas perturber les élèves. L'analyse des résultats est pourtant à nuancer. Pour les élèves qui ont réalisé l'exercice (les deux élèves précédemment cités, en situation de handicap, n'ont pas réussi à placer les poubelles), deux stratégies s'individualisent : ceux qui ont pris en compte les axes de circulation (les flux) et ceux qui ont réparti de façon équitable les cubo'verres. Dans ce cas de figure, il est difficile de repérer s'ils manipulent la maille ou l'« égalité » territoriale, soit plutôt la distance que la maille.

Dans un second temps, par groupe de quatre, en phase collective, la même consigne est rappelée. Les élèves doivent s'accorder, revenir (ou pas) sur les localisations initiales et personnelles, afin de produire une nouvelle proposition. A l'issue de cette nouvelle phase, huit groupes sur huit proposent d'installer les cubo'verres sur des axes ou lieux fréquentés. Aucun des huit groupes ne positionne des cubo'verres sur des zones vides, ni ne positionne des cubo'verres les uns à côté des autres. Deux groupes oublient des lieux de fortes densités, mais trois autres groupes leurs attribuent plusieurs gommettes. Les groupes d'élèves ont fait le lien entre la densité de population et donc des besoins en cubo'verres plus important Ils vont au-delà des facteurs explicatifs proposés. En conclusion, pour aucun des groupes, la localisation des PAV ne relève du hasard ou d'un raisonnement géographique erroné. 
Figure 5 : exemple de plan complété par un groupe (Servais 2015)

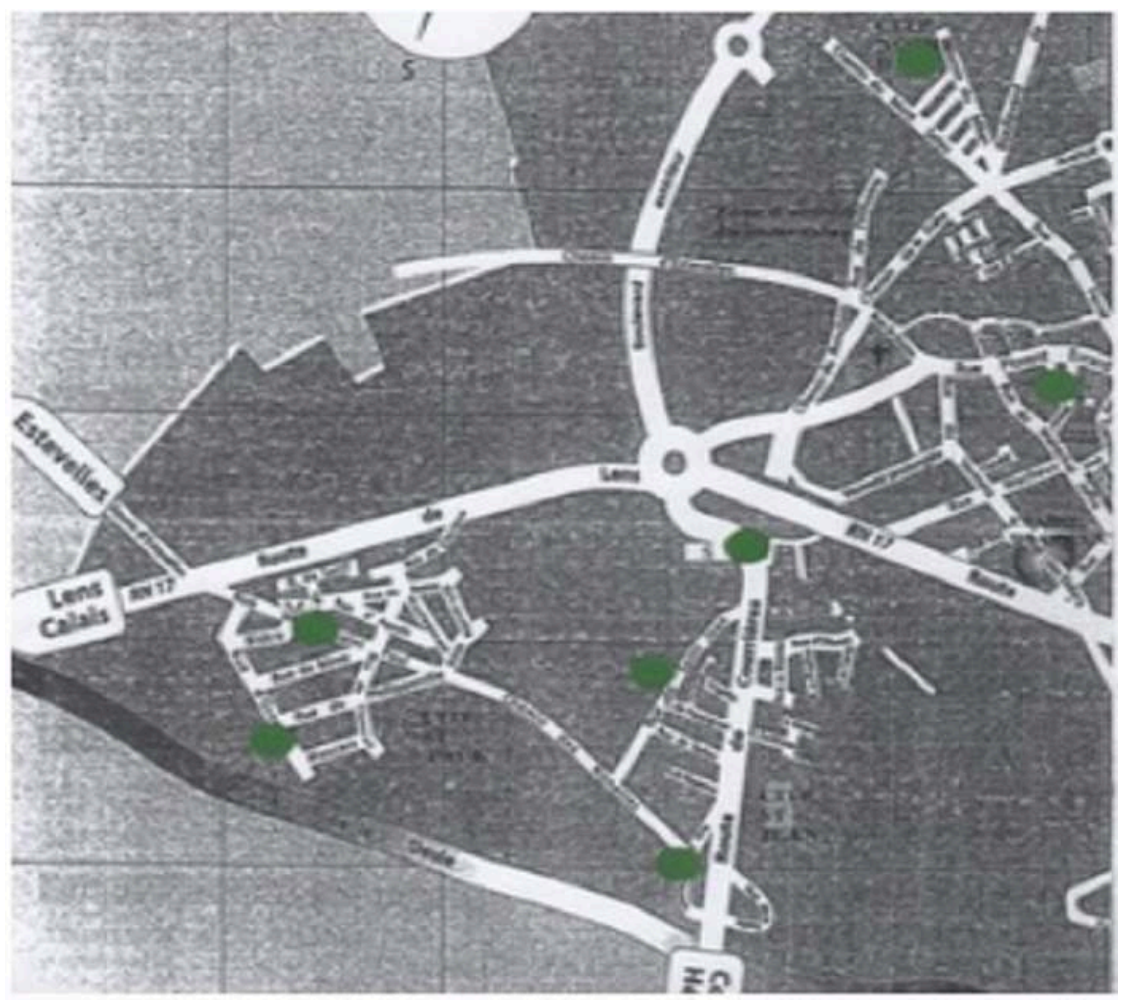

Figure 6 : extrait de la carte du quartier de l'école (Servais 2015)

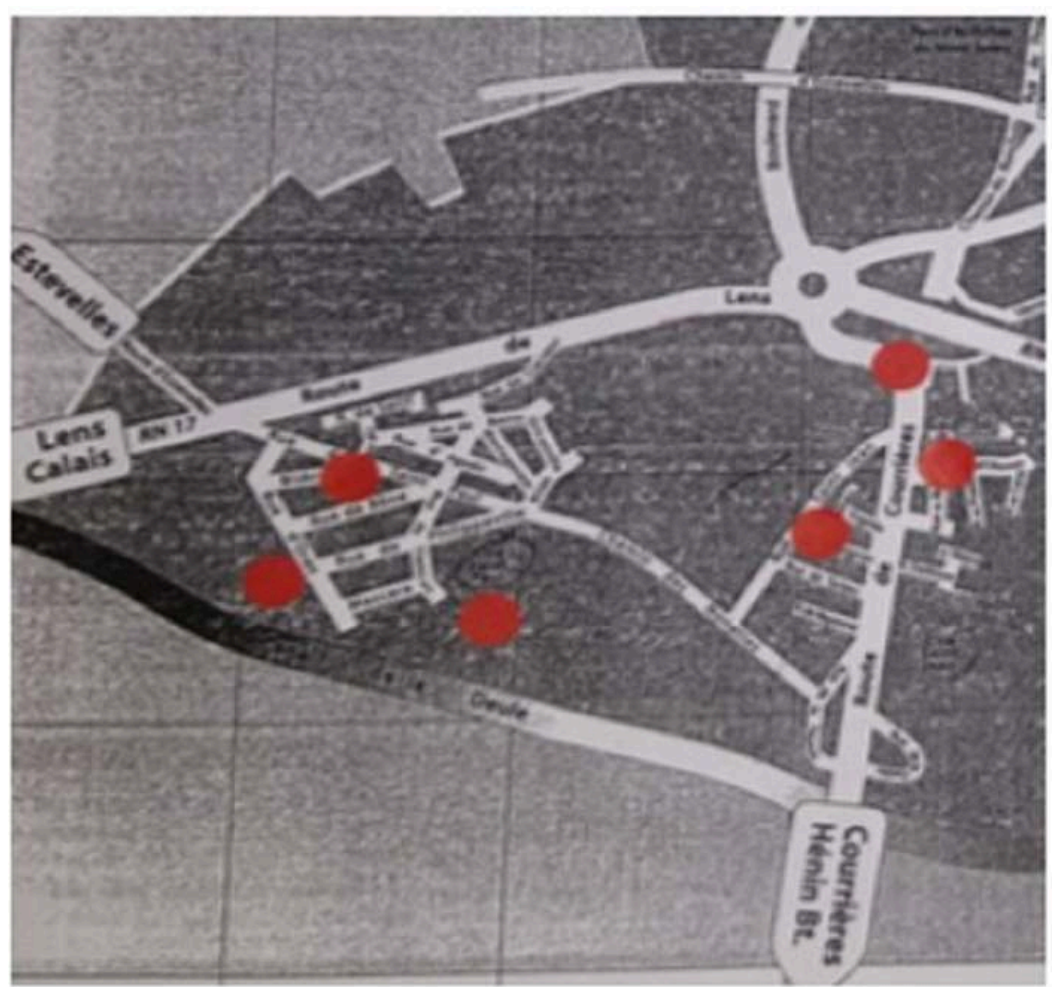

Lors de cette confrontation de points de vue, prendre une décision à plusieurs sur l'aménagement d'un espace nécessite de la justifier par la prise en compte des lois de 
l'espace manipulées lors de la séance précédente. A l'issue de la phase de recherche, la mise en commun est menée à l'oral. Les échanges proposent des arguments qui sont cohérents. La quasi-totalité des élèves est en capacité de prendre en compte le changement d'échelle et de la taille du nouvel espace et donc, de la nécessité de multiplier les cubo'verres (contrairement à la classe où une seule poubelle suffisait). Ils prennent en compte la complexité des facteurs : densité de population, hiérarchies des axes de communication, positionner les cubo'verres sur les axes très fréquentés (ce qui induirait que certains élèves se sont correctement situés et ont repéré les axes sur le plan). La compréhension des enjeux spatiaux, voire territoriaux est bien en construction chez les élèves. Le seul biais est que l'expérimentation ne permet pas une différenciation précise des élèves.

La séance 3, qui dure une heure, propose d'analyser la position effective des cubo'verres (carte réalisée par l'enseignante qui a collecté les informations auprès de la communauté de communes et a réalisé la carte ci-dessous)

Figure 7 : localisation des Cubo'verres de la commune (Servais, 2015)

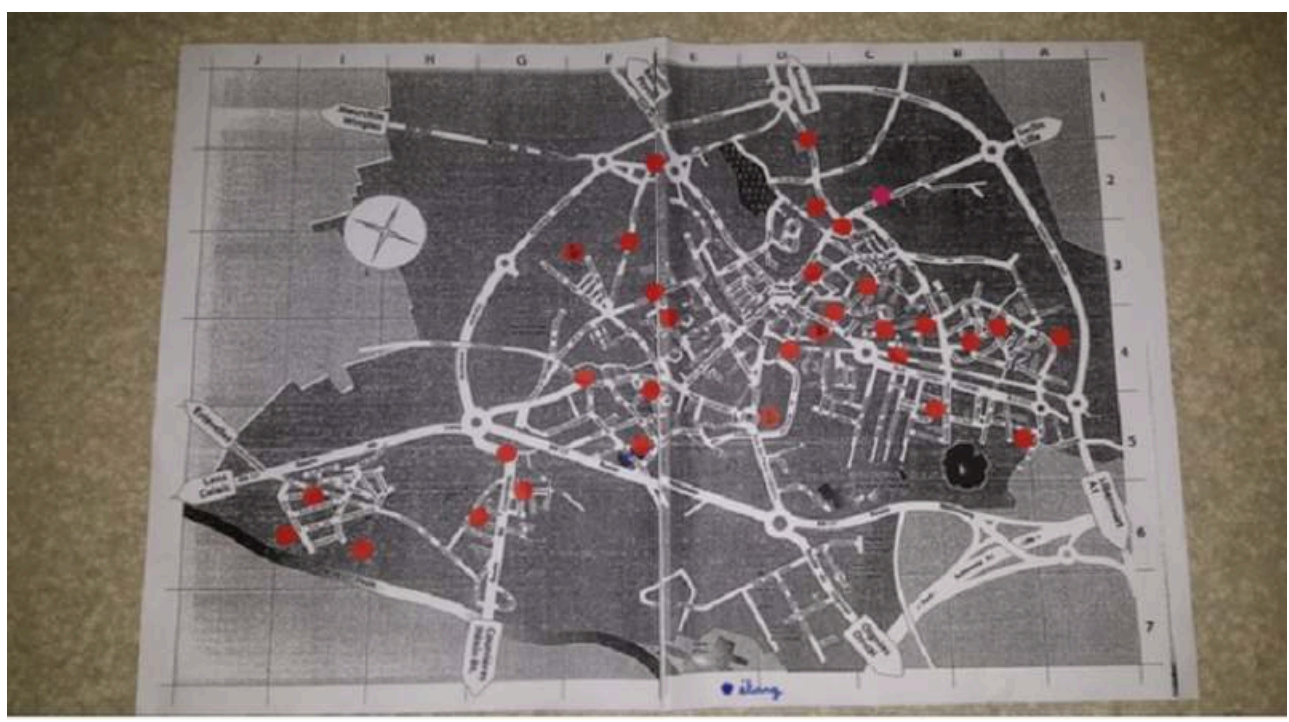

L'objectif est une évaluation formative. Les élèves doivent vérifier que l'ensemble des cubo'verres de la ville est correctement positionné et donc si les choix du maire sont bons. Ils doivent choisir les bons arguments pour valider ou non la localisation des cubo'verres. Ainsi, les élèves repèrent l'absence de cubo'verres au Sud de la ville et s'interrogent sur ce fait. Ils émettent l'hypothèse d'un quartier peu peuplé. On constate donc que le raisonnement géographique élaboré par les élèves est plus complexe que la prise en compte des deux notions (maille et flux) et qu'ils prennent vraisemblablement en compte la densité de population. Ceci pourrait permettre de lever le doute sur le fait que la complexité (et la pensée systémique, soit les interactions entre les facteurs) ne serait pas un obstacle à l'apprentissage.

\section{Conclusion}

31 Notre échantillon d'étude nous a donné une première idée de la diversité de l'utilisation des jeux en cours de géographie. Nous avons constaté parfois un écart entre les ambitions affichées par les concepteurs de jeu et les attentes des programmes 
et de leurs objectifs citoyens, même si, nous l'avons précisé, certains jeux ont plus de 10 ans d'existence. La motivation semble souvent avoir été un critère parfois exclusif, permettant de pallier un enseignement de la géographie peu attractif pour les élèves (discipline où la mémorisation peut être considérée comme l'activité première). Les jeux proposés sont alors adaptés de jeux de société. Aujourd'hui, des enseignants proposent d'autres catégories de jeu qui offrent des objectifs d'apprentissage didactiquement recevables.

La jeune enseignante, à l'initiative du jeu T'es ou cubo ?, en a tiré de nombreux intérêts dont celui de prendre goût à l'enseignement de la géographie. Ce qui devrait rassurer les enseignants à pratiquer une géographie complexe et systémique, puisque quel que soit l'âge (ou presque), des enfants pourraient jouer pour apprendre. L'entrée du jeu dans la classe a été progressive et il est normal que les enseignants s'appuient sur des références connues (Sept familles, Trivial Pursuit) pour tester de nouvelles pratiques. Mais, il est intéressant de noter que la pratique du jeu peut remettre en cause la vulgate pour enseigner une géographie plus ambitieuse. C'est-à-dire, répondre aux injonctions des programmes de 2015, «l'enseignement de la géographie aide l'élève à penser le monde. Il lui permet aussi de vivre et d'analyser des expériences spatiales et le conduit à prendre conscience de la dimension géographique de son existence. Il participe bien de la construction de l'élève en tant qu'habitant. » Ainsi, à partir de cas très concrets ou de jeux, les élèves pourraient percevoir la complexité du monde, leur rôle dans les sociétés et comment celles-ci perçoivent, organisent leurs territoires.

L'objectif est bien sûr de poursuivre cette exploration de jeux destinés à la géographie pour enrichir une typologie qui demeure aujourd'hui au stade de la préfiguration, même si les bases sont posées. Ce que nous espérons, par l'outil proposé, cette typologie qui se veut pragmatique, c'est qu'il encourage la fertilisation croisée des choix (pédagogique et didactique) afin de mettre en lumière l'émergence possible d'une formation à la compréhension des enjeux territoriaux et d'amener les élèves à devenir des acteurs (voire des experts) de leur territoire.

\section{BIBLIOGRAPHIE}

BROUGÈRE G. (1995), Jeu et éducation, Paris, L'Harmattan.

FONTANABONA J., THEMINES J.F. (dir.) (2005), Innovation et histoire-géographie dans l'enseignement secondaire. Analyse didactiques, Lyon, Institut national de recherche pédagogique

GRATALOUP Ch. (1989) dans La géographie dans tous ses états, Paris, CNDP

JACOB F. (2005), « “Geographic poubelle situation (GPS)”. Jeu d'aménagement et jeu de rôle en classe de $6^{\mathrm{e}}$ ", in Masson-Vincent M. (dir.), Jeu, géographie et citoyenneté. De l'école à l'université, Paris, Seli Arslan

LE ROUX, A. (2003 [1997]), Didactique de la géographie, Caen, Presses universitaires de Caen. 
MASSON VINCENT M. (1994), Vous avez dit géographies? Didactique d'une géographie plurielle, Paris, A.Colin.

MASSON VINCENT M. (dir.), (2005), jeux, géographie et citoyenneté de l'école à l'Université, Paris, Seli Arslan

MERENNE-SCHOUMAKER B. (2005), Didactique de la Géographie. Organiser les apprentissages, Louvain-la- Neuve, De Boeck.

MUSSER Marie et THIBERT Rémi (2009), Quelles relations entre jeu et apprentissages à l'école? Une question renouvelée. Dossier d'actualité de la VST, $\mathrm{n}^{\circ}$ 48, http://www.inrp.fr/vst/LettreVST/ 48-octobre-2009.php.

PACHE A., HERTIG P. CURNIER D. (2016), « Approches de la complexité dans le contexte de l'éducation en vue du développement durable : quelles perspectives pour la didactique de la géographie ", Les sciences de l'éducation pour l'Ere nouvelle, vol. 49, n 4, pp. 15-45

POTIER V. (2016), « “Soyons sérieux et jouons un peu!” Navigation aux frontières de la classe par le jeu vidéo d'apprentissage Mecagenius. », Sciences du jeu n 5, http://journals.openedition.org/ sdj/638

OFFNER J.M.(2013), « Flux », in J. Lévy et M. Lussault (dir.), Dictionnaire de la géographie et de l'espace des sociétés, Belin, Paris, pp. 398-400.

RIMBERT S. (1998), « Supprimons la géographie ! », Cybergeo, http://journals.openedition.org/ cybergeo/5537

SERVAIS E. (2015), « Quand Éducation au Développement Durable et aménagement de l'espace ne font qu'un. Former le citoyen aux enjeux territoriaux », Mémoire de Master 2 MEEF - mention premier degré, ESPE Lille Nord de France

THEMINES J.F. (2004), Quatre conceptions de la géographie scolaire : un modèle interprétatif des pratiques d'enseignement de la géographie ?", Cybergéo, document 262, http:// journals.openedition.org/cybergeo/4325

\section{NOTES}

1. BOEN spécial n² du 26-3-2015

2. http://eduscol.education.fr/jeu-numerique/

3. www.lepetitjournaldesprofs.com/reseauludus/

4. BOEN spécial $n^{\circ} 11$ du 26 novembre 2015

5. X. Leroux et C. Leguen http://didageo.blogspot.fr/2016/12/le-raisonnement-engeographie-dans-les.html

\section{RÉSUMÉS}

Jouer en cours de géographie n'est plus à proprement parler une innovation. Le jeu des villes de Grataloup, édité en 1989, a bientôt trente ans ! Ce faisant, les jeux offrent un champ des possibles 
tout à fait recevable, répondant aux objectifs actuels des programmes et des recherches. Ils pourraient avoir une place importante (en aucun cas exclusive) pour redonner du sens à l'enseignement de la géographie. Ainsi, de connaissances apprises (et vite oubliées) à des savoirs construits par les élèves, des notions manipulées, le jeu a toute sa place dans l'enseignement de la géographie. A partir des jeux du site réseau Ludus, cadre d'un premier état des lieux amené à être enrichi, cet article propose une analyse critique des objectifs d'enseignement. Par le truchement d'une grille d'analyse, il repère de premières tendances afin d'initier une réflexion de ce que les jeux pourraient offrir en matière de renouvellement de l'enseignement de la géographie, notamment pour penser la compréhension des enjeux spatiaux.

Learning through play during geography lessons is no more an innovative process. Indeed the game 'Jeu des villes' by Grataloup was edited in 1989, nearly 30 years ago. In so doing, games offer a wide choice of possibilities meeting all educational curriculums and research programs. Games could play an important role in giving a new meaning to geography lessons. So from learned knowledge (soon forgotten) to knowledge built by pupils, playing in classes has a special and necessary place. This article proposes to analyze educational goals through games founded on the web site Réseau Ludus. Thanks to an evaluation grid, the first tendencies show how games could provide the renewal of educational geography goals, especially by rethinking how to understand space-related issues.

\section{INDEX}

Mots-clés : jeu, didactique de la géographie, raisonnement, géographie rénovée, enjeux territoriaux

Keywords : game, didactic, reasoning, new geography, territorial issue/challenge

\section{AUTEURS}

\section{FRÉDÉRIQUE JACOB}

ESPE Lille Nord de France - Cirel Lille 3

\section{EMILIE SERVAIS}

Professeur des écoles - Académie de Lille 